\title{
LETTER
}

\section{Passive immunotherapy of sepsis with intravenous immune globulin: not all IVIg preparations are created equal}

\author{
Tchavdar Vassilev* and Michael Bauer
}

Normal intravenous immunoglobulin (IVIg) preparations are known to neutralize a wide array of pathogens plus their virulence factors and in addition possess broad antiinflammatory and immunomodulating activities. However, these preparations have not been really successful in their present form in preventing sepsis-related death. The recent report by the International Neonatal Immunotherapy Study Collaborative Group is the account of the last in a series of clinical trials that have failed to show an effect of IVIg administration on sepsis outcome [1]. Each batch of IVIgs is produced by fractionation of a plasma pool obtained from at least 10,000 healthy donors. All these studies have used different immunoglobulin preparations assuming that their properties are identical as the IgG repertoire in them is representative for a large population of healthy plasma donors.

Licensed IVIg preparations are, however, not equal. One should keep in mind that their antigen-binding behavior and biological properties depend on the fractionation technology used to manufacture them. Therapeutic immunoglobulins are very polyspecific. Our previous studies have shown that the preparations produced using a protein fractionation step at low $\mathrm{pH}$ have an additionally enhanced polyspecificity. They possess higher functional antigen-binding affinity and bind to an increased spectrum of bacterial antigens. Interestingly, the exposure to low $\mathrm{pH}$ also results in acquiring the ability to interact with a pro-inflammatory cytokine (IFNY) and to inhibit strongly phytohemagglutinin-induced human T-cell proliferation. While the passive immunotherapy of mice with bacterial lipopolysaccharide-induced systemic inflammation and shock with a single dose of the native IVIg (not exposed to $\mathrm{pH}$ 4.0 buffer) had no effect on mortality, the same preparation subjected previously to low $\mathrm{pH}$ conditions significantly increased their survival $[2,3]$.

*Correspondence: tchavdarv@gmail.com

Center for Sepsis Control and Care, University Hospital, Friedrich Schiller University, Erlanger Allee 101, 07747 Jena, Germany
Some experimental IVIg preparations possess an even stronger polyspecificity. A recent study has shown that a single dose of Fe(II)-exposure-modified IVIg prevented the death of mice with experimental septic shock, induced by the injection of bacterial lipopolysaccharide or of live Escherichia coli, while the native preparation failed to do so $[4,5]$. Antibodies of the IgM isotype are inherently polyspecific and thus IgM-enriched immunoglobulins should also have a strong therapeutic potential. In addition to sepsis, the passive immunotherapy with modified intravenous immunoglobulins could also be beneficial in other variants of the severe inflammatory response syndrome (post-traumatic, after severe burns, in avian flu, and so on). While the IgM-enriched and the ferrous ion exposure-modified IVIg are experimental drugs, several acid $\mathrm{pH}$-modified IVIg preparations are already licensed, commercially available and should be considered as an adjunctive treatment in sepsis patients.

\section{Abbreviations}

IVlg, intravenous immunoglobulin.

Competing interests

TV has a patent application describing the production of a modified immunoglobulin preparation. MB declares that he has no competing interests. Published: 25 January 2012

\section{References}

1. The INIS Collaborative Group: Treatment of neonatal sepsis with intravenous immune globulin. NEng/ J Med 2011, 365:1201-1211.

2. Djoumerska IK, Tchorbanov Al, Pashov AD, Vassilev TL: The autoreactivity of intravenous immunoglobulin (IVIg) preparations depends on the methods for fractionation. Scand J Immunol 2005, 61:357-363.

3. Djoumerska-Alexieva IK, Dimitrov JD, Voynova EN, Lacroix-Desmazes S, Kaveri SV, Vassilev TL: Exposure of IgG to an acidic environment results in molecular modifications and in enhanced protective activity in sepsis. FEBS J 2010, 277:3039-3050.

4. Dimitrov JD, Ivanovska N, Lacroix-Desmazes S, Doltchinkova VR, Kaveri SV, Vassilev TL: Ferrous ions and reactive oxygen species increase antigenbinding and anti-inflammatory activities of immunoglobulin G.J Biol Chem 2006, 281:439-446.

5. Djoumerska-Alexieva I, Dimitrov J, Nacheva J, Kaveri S, Vassilev T: Proteindestabilizing agents induce polyreactivity and enhanced immunomodulatory activity in IVIg preparations. Autoimmun 2009, 43:365-367.

\section{doi:10.1186/cc10607}

Cite this article as: Vassilev T, Bauer M: Passive immunotherapy of sepsis with intravenous immune globulin: not all IVIg preparations are created equal. Critical Care 2012, 16:407. 